\title{
RECENT OPTICAL OBSERVATIONS OF SUPERNOVAE
}

\author{
Alexei V. Filippenko \\ Department of Astronomy \\ University of California \\ Berkeley, CA 94720 \\ USA
}

\begin{abstract}
This paper reviews some of the basic optical properties of supernovae (SNe), concentrating on the most interesting new observations. It is emphasized that $\mathrm{SNe}$ can be securely subdivided into the two well-known classes (Type I and Type II) only on the basis of their spectra, since the light curves alone can be misleading. Brief interpretations of the spectra and light curves are presented. Spectroscopic and photometric characteristics of "peculiar" Type I supernovae (SNe I-pec), which may be physically distinct from "normal" SNe I, are discussed. Finally, the complete optical spectrum of SN 1985f in NGC 4618 is illustrated, and comparisons are made with the spectra of SNe I and SNe II. No resemblance is found, suggesting that this object belongs to a truly new class (Type III) of SNe. There is, however, very recent evidence that the spectra of SNe I-pec obtained more than eight months past maximum brightness are strikingly similar to that of SN $1985 f$.
\end{abstract}

\section{INTRODUCTION}

Supernovae are exploding stars whose brightness can rival that of their host galaxies for a short period of time ( $\lesssim 1$ month). A majority of these objects have been placed into two categories that are distinguished primarily by the appearance of their optical spectra. Type II supernovae (SNe II) exhibit broad Balmer lines, while those of Type I (SNe I) do not. Their optical light curves also differ, but an unambiguous distinction cannot always be made. If $H_{0}=50 \mathrm{~km}$ $\mathrm{s}^{-1} \mathrm{Mpc}^{-1}$, then SNe I all have $M_{V} \approx-20$ at maximum light, and may be useful as standard candles. SNe II are perhaps a magnitude fainter, and show greater intrinsic scatter in their photometric and spectroscopic characteristics. SNe I occur in all kinds of galaxies, whereas SNe II appear almost invariably in the arms of spirals. It is generally believed that the physics governing the explosions is different in the two types, and that the progenitors of SNe I are low-mass stars $\left(M \approx 1 M_{\odot}\right)$ while those of SNe II are quite massive $\left(M \gtrsim 8 M_{\odot}\right)$. There is, however, evidence that some SNe I are associated with massive stars. Moreover, as will be shown in §4, at least one other type of SN which is fundamentally different from classical SNe probably exists. A recent review by Panagia (1986) may be consulted for information on topics only briefly addressed here. 


\section{SPECTROSCOPIC PROPERTIES OF SNe I and SNe II}

Spectra of SN 1937c in IC 4182, obtained and analyzed by Minkowski (1939), effectively define what is meant by SNe I, although more modern, photometrically calibrated data (e.g., SN 1972e in NGC 5253: Kirshner et al. 1973) are now of greater value in making direct comparisons. The optical spectra of normal SNe I are all very similar, and in no cases have lines of $\mathrm{H}$ been detected. Unlike SNe I, SNe II show considerable variety in their spectroscopic characteristics, although Balmer lines (sometimes having $\mathrm{P}$ Cygni profiles) are always present. Lines of He I have also been seen, but not in SNe I.

A good example of a Type I object is SN 1983r in IC 1731. Figure 1 illustrates seven spectra of this object, ranging from about two to four weeks past maximum brightness. These were obtained with the $1.5-\mathrm{m}$ reflector at Palomar

SN $1983 \mathrm{r}$ in IC 1731

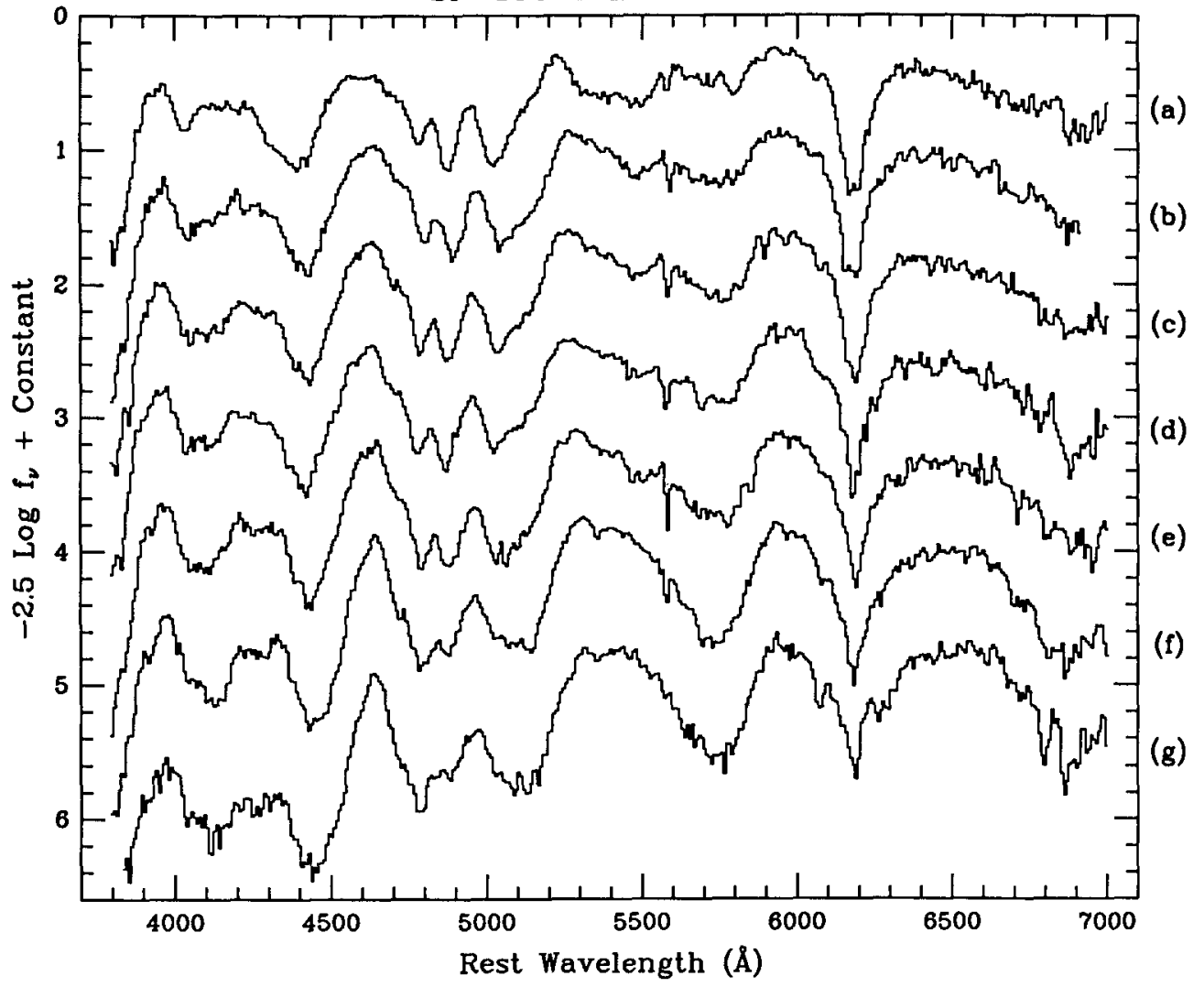

Figure 1: Seven spectra of SN 1983r in IC 1731 (with arbitrary additive offsets) show features typical of SNe I, including a prominent absorption line at $\sim \lambda 6180$. Note the absence of hydrogen. The UT dates are $6,9,10,11,12,16$, and 18 October 1983 for $(a)$ through $(g)$. Maximum light was probably in late September 1983. Incompletely subtracted [O I] from the night sky is responsible for the weak, narrow feature at $\sim \lambda 5577$, while telluric $\mathrm{O}_{2}$ produces the dip near $\lambda 6860$. 
Observatory (Porter 1983) and kindly made available by A. C. Porter of Caltech. The prominent dip at $\sim \lambda 6180$ is one of the most important and persistent features of normal SNe I; indeed, it is often the main secondary diagnostic in the classification of SNe. If it is produced by blueshifted Si II $\lambda 6355$, as is generally believed, then expansion velocities of $\gtrsim 8000 \mathrm{~km} \mathrm{~s}^{-1}$ are derived for the lineproducing photosphere. Note that the line becomes narrower and fades with time, but new ones at $\sim \lambda 6080$ and $\sim \lambda 6260$ maintain the strength of this trough. Other features of interest include the prominent trough at $\sim \lambda \lambda 5500-5900$, which steadily grows as the SN ages, and the strong emission at $\lambda 4600$, which gets narrower and slowly shifts toward longer wavelengths. As in other SNe I, the blue end of the continuum fades faster than the red.

Spectra of SNe I spanning a wide temporal baseline have been presented by Minkowski (1939), Kirshner et al. (1973), Branch et al. (1983), De Robertis and Pinto (1985), and others. During the first few months after maximum light, the spectra are dominated by $\mathrm{P}$ Cygni features superposed on a thermal continuum which carries the bulk of the radiated flux. These lines are probably produced by permitted transitions of singly ionized metals (especially $\mathrm{Fe}$ ), but synthetic spectra are necessary to help establish line identifications and to accurately reproduce the observations (Branch 1982). It is assumed that lines are formed by resonance scattering above a sharp photosphere; the emission peaks therefore appear at approximately the rest wavelengths, while the absorption components are blueshifted by the velocity of the photosphere. At late times, on the other hand, the spectra may be dominated by thousands of forbidden emission lines of Fe II, Fe III, and Co III in an essentially optically thin nebula (Axelrod 1980). As noted by De Robertis and Pinto (1985), this is rather puzzling because the general appearance of the spectra does not change much with time. The effects of absorption in the emission-line model are currently being investigated to help clarify the connection between the early and late-time spectra.

Figure 2 shows the spectrum of the Type II SN 19851 in NGC 5033, obtained with the Palomar 5-m telescope several weeks after maximum (Filippenko and Sargent 1986b). Somewhat blueshifted emission lines of $\mathrm{H} \alpha$ and $\mathrm{H} \beta$ dominate the spectrum, but there are also strong absorption lines. Several features undoubtedly have P Cygni profiles. Unlike most other SNe II, SN 1985l exhibits strong absorption at $\sim \lambda 6210$, which may be the blueshifted Si II $\lambda 6355$ line generally associated with SNe I. This is only one example of the substantial inhomogeneity among spectra of SNe II.

Long-term studies of SNe II such as those published by Kirshner et al. (1973), Kirshner and Kwan (1974), and Branch et al. (1981) show that within a few months past maximum light, the spectra are generally dominated by a strong blue continuum having a roughly blackbody shape $(T \approx 5000-10000$ K). Weak spectral lines that strengthen with time are also visible. Most have $\mathrm{P}$ Cygni profiles which are produced by resonance scattering in an expanding atmosphere. $\mathrm{H} \alpha$, on the other hand, often does not exhibit a $\mathrm{P}$ Cygni profile, so collisional excitation may be responsible for the net emission in this line and in several others as well (Kirshner and Kwan 1975; Branch et al. 1981). New aborption and emission lines appear and grow as the $\mathrm{SN}$ ages; these include $\mathrm{Ca}$ II $\mathrm{H}$ and $\mathrm{K}$, the infrared triplet of $\mathrm{Ca}$ II, $\mathrm{Na} \mathrm{I} \mathrm{D,} \mathrm{Mg} \mathrm{I} \mathrm{b,} \mathrm{and} \mathrm{lines} \mathrm{of} \mathrm{Fe} \mathrm{II} \mathrm{and}$ He I. $\mathrm{H} \alpha$ dominates the spectrum after a month or two past maximum, and the blue continuum fades considerably during this time. At late stages ( $\gtrsim 4$ months past maximum), emission lines of [O I] $\lambda \lambda 6300,6364$ and [Ca II] $\lambda \lambda 7292,7324$ are visible. 


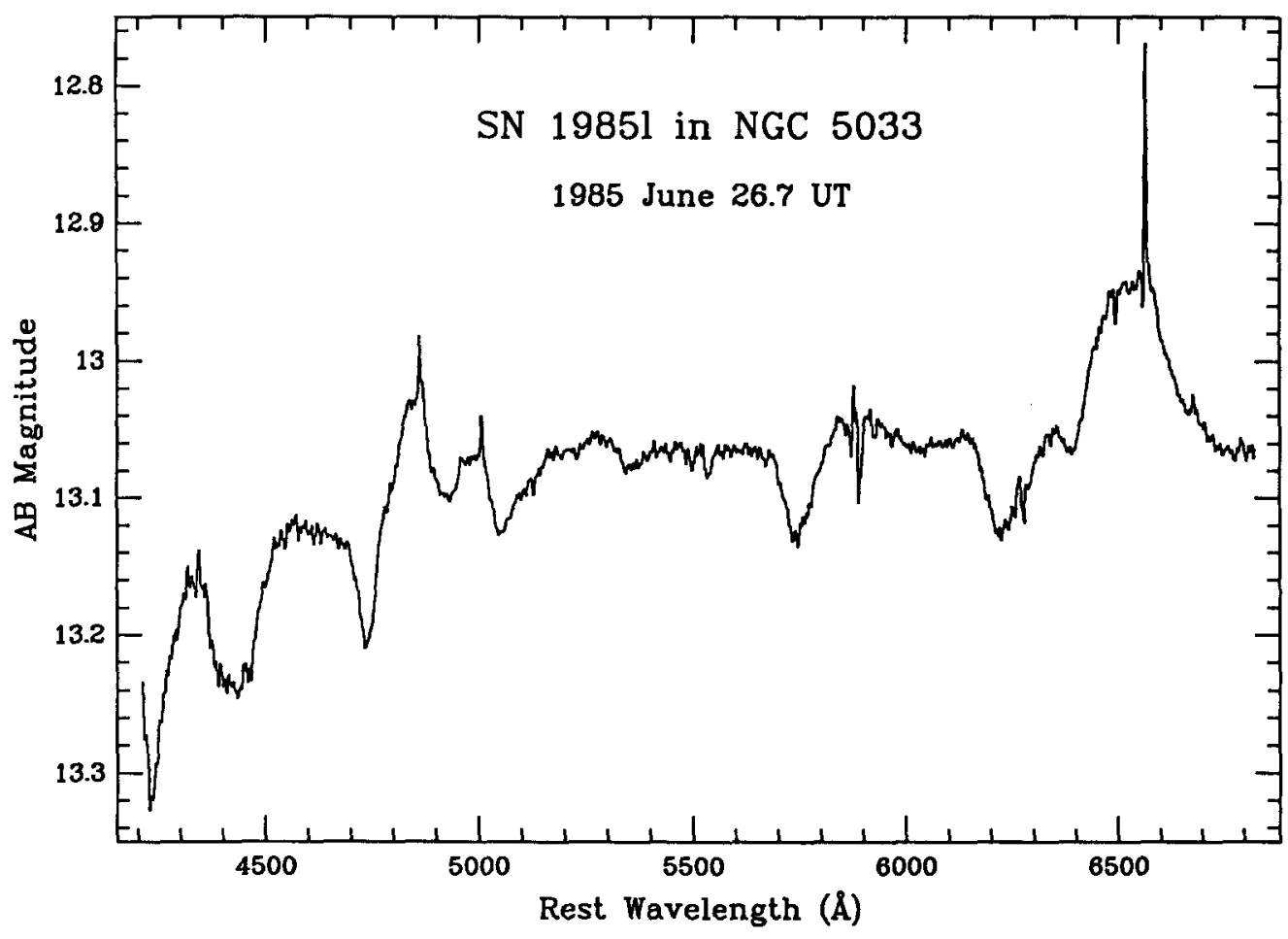

Figure 2: Spectrum of SN 19851 in NGC 5033 several months after maximum brightness. Magnitude $\mathrm{AB}=-2.5 \log _{10} f_{\nu}-48.6$, where the units of $f_{\nu}$ are erg $\mathrm{s}^{-1} \mathrm{~cm}^{-2} \mathrm{~Hz}^{-1}$. Prominent Balmer emission lines indicate that this is a Type II SN, but the absorption at $\sim \lambda 6210$ may be blueshifted Si II $\lambda 6355$, which is normally seen in SNe I. Some of the lines clearly have P Cygni profiles. Narrow emission ( $\mathrm{H} \alpha, \mathrm{H} \beta,[\mathrm{O} \mathrm{III}])$ is produced by $\mathrm{H}$ II regions.

The only high-quality spectra of SNe II that have been obtained prior to maximum light are those of SN 1983k in NGC 4699.(Niemela, Ruíz, and Phillips 1985). They showed emission lines of H I, He II, and N III superposed on a very blue continuum. These lines disappeared near maximum, leaving weak absorption lines of $\mathrm{H} \mathrm{I}, \mathrm{He} \mathrm{I}$, and $\mathrm{Ca}$ II, and a month later the spectra resembled those of previously studied SNe II. The absorption lines at maximum, as well as the extended peak in the light curve, imply that the progenitor had an extensive, preexisting circumstellar shell. It is therefore likely to have been a red supergiant or a Wolf-Rayet star, especially since its surface layers were overabundant in nitrogen.

\section{PHOTOMETRIC PROPERTIES OF SNe I and SNe II}

Long ago astronomers recognized that the two main types of SNe seem to have different light curves, and at times the light curve alone is used to classify objects for which spectra are not available. Many authors (e.g., Zwicky 1965; Oke 
and Searle 1974) caution that this is a risky procedure which can only result in confusion; it assumes a tight correlation between spectrum and light curve that has not been firmly established by observations.

It is, of course, extremely important to study the light curves of objects for which spectroscopic classifications exist, since they may provide valuable clues to the physical conditions and processes in SNe. A recent paper by Doggett and Branch (1985, hereafter referred to as DB85) presents direct comparisons between the shapes of light curves for different types of SNe. Barbon, Ciatti, and Rosino $(1973,1979)$ had previously compiled most of the blue magnitudes used by DB85, but the far less extensive visual data were taken from a variety of sources in the literature.

The composite light curve for SNe I exhibits a relatively small amount of scatter, confirming the good photometric homogeneity of this class. After a rapid rise over $\sim 15$ days prior to maximum light $(t=0)$, the brightness decreases by $\sim 2.8 \mathrm{mag}$ during the following 40 days. A distinct "elbow" occurs at $\sim 35$ days past maximum, after which SNe I fade more slowly, but another, much less noticeable bend is visible about 65 days later. Between 100 and 400 days past maximum, the curve declines at a constant rate of $\sim 0.016 \mathrm{mag} \mathrm{day}^{-1}$.

Although DB85 treat SNe I as a single uniform class, Barbon, Ciatti, and Rosino (1973), Barbon (1978), and others have shown that the light curves fall into two rather distinct subdivisions known as "fast" and "slow." The width of the light curve 2 mag below maximum is 32 days in "fast" SNe I, and 38 days in the "slow" variety. Moreover, the amplitude from the maximum to the "elbow" at $t \approx 35$ days is $3.25 \mathrm{mag}$ and $2.50 \mathrm{mag}$ in the "fast" and "slow" light curves, respectively. The corresponding rates of decline in the exponential tail are $\sim 0.016$ and $0.012 \mathrm{mag} \mathrm{day}^{-1}$. Finally, there may be spectroscopic differences between these two subclasses of SNe I (see \$4).

The light curves of SNe II can also be divided into two fairly homogeneous subclasses, but these differ from each other much more than the "fast" and "slow" SNe I (Barbon, Ciatti, and Rosino 1979). They are labeled "plateau" (SNe II-P) and "linear" (SNe II-L), and composites of many SNe II of each subclass are illustrated by DB85. The SNe II-L light curves resemble those of SNe I, but their initial decline after maximum is not as rapid as in $\mathrm{SNe} I$, and the decline in the exponential tail $\left(t \gtrsim 100\right.$ days) is slower $\left(0.012 \mathrm{mag} \mathrm{day}^{-1}\right)$. SNe II-P, on the other hand, exhibit a rather slow decline past maximum, followed by a phase of nearly constant brightness which constitutes the "plateau." After $t \approx 60$ days, the brightness declines once again, and the slope changes several times. At late times ( $t \gtrsim 200$ days), the decline is given by $0.0075 \mathrm{mag}^{-1} \mathrm{day}^{-1}$. DB85 claim that it is safe to classify a SN as Type II-P on the basis of the light curve alone, because the "plateau" phase is so distinctive. They emphasize, however, that the light curves of SNe I and SN II-L are so similar to each other that one cannot unambiguously distinguish between them in a given supernova, considering that individual objects can show significant departures from the mean curves. Classification based on partial light curves is especially suspect.

As discussed by DB85, differences in the light curves of SNe II-P and SNe II$L$ may be due primarily to variations in the mass of the hydrogen envelope which is believed to be ejected during the collapse of the heavy-element core in a star having $M \gtrsim 8 M_{\odot}$. If the progenitors of SNe II-L somehow lose a large portion of their envelope prior to the explosion, the plateau should be much shorter (and perhaps even absent) since it is normally caused by the diffusive release of shock 
energy from the massive expanding envelope (e.g., Schurmann, Arnett, and Falk 1979; Litvinova and Nadyozhin 1983). If so, the light curves of SNe II should show a continuous range of shapes.

It is also possible, however, that SNe II-L may be related to SNe I in a fundamental manner. Perhaps the progenitors of SNe II-L have $M \lesssim 8 M_{\odot}$ and lose most, but not all, of their hydrogen envelopes before igniting their degenerate carbon cores. The optical spectra would then reflect their $\mathrm{H}$-rich envelope, but the physics of the explosion would be more similar to that of SNe I than SNe II-P. Since a substantial amount of $\mathrm{Ni}^{56}$ should be produced in the core, normal beta decay (to $\mathrm{Co}^{56}$, and then to stable $\mathrm{Fe}^{56}$ ) would account for the shape of the light curve in the exponential tail, as in SNe I.

\section{PECULIAR TYPE I SUPERNOVAE, AND SN $1985 f$ IN NGC 4618}

One of the principal features in the optical spectra of normal SNe I near maximum light is the absorption line at $\sim \lambda 6150$, thought to be blueshifted Si II $\lambda 6355$. A number of SNe I, on the other hand, do not exhibit this feature; instead, there may be an absorption-line "doublet" at $\sim \lambda 6300$ and $\lambda 6500$. Examples of these "peculiar Type I supernovae" (SNe I-pec) include SN 1962l in NGC 1073 (Bertola 1964), SN 19641 in NGC 3968 (Bertola, Mammano, and Perinotto 1965), SN 1983n in NGC 5236 (Uomoto and Kirshner 1985), and SN 19841 in NGC 991 (Wheeler and Levreault 1985). SNe I-pec are $~ 1.5$ mag fainter than normal SNe I. Their optical light curves are of the "slow" type described in $\S 3$, but they can be unambiguously distinguished from normal SNe I by their infrared light curves (Elias et al. 1985). It appears that their spectra near maximum resemble those of SNe I one to two months past maximum. They are also redder than typical SNe I. Finally, all occur in late-type (Sc) galaxies, and usually in prominent H II regions.

The fact that SNe I-pec can easily be mistaken for classical SNe I, but are roughly a factor of 4 fainter, means that some contamination of the sample of SNe I used to calibrate the extragalactic distance scale may occur (Uomoto and Kirshner 1985). Careful spectroscopy is therefore necessary in all cases except those for which high-quality infrared light curves in the properly redshifted bands are available.

There are now good reasons to believe that SNe I-pec represent a fundamentally new subclass of supernovae. Wheeler and Levreault (1985), for example, have argued that these objects eject less radioactive $\mathrm{Ni}^{56}$ than normal $\mathrm{SNe} \mathrm{I}$, but a similar total mass. If so, they derive most of their kinetic energy from core collapse rather than from thermonuclear burning. Their progenitors are probably quite massive stars $\left(M \approx 10-20 M_{\odot}\right)$ which explode in much the same way as SNe II. Since Balmer lines are not seen in the spectra, prior to the explosions the progenitors must have lost their hydrogen envelopes by winds, binary mass transfer, or some other mechanism. Chevalier (1986) has recently argued that Wolf-Rayet stars are likely candidates.

On 28 February 1985 UT, Filippenko and Sargent (1985) discovered what appears to be yet another "peculiar" type of supernova (SN 1985f), but its spectral characteristics are unprecedented (Fig. 3). Strong, very broad emission lines similar to those in quasars but having the wrong relative wavelengths are superposed on a continuum whose slope is roughly linear in a plot of $f_{\lambda}$ versus $\lambda$. There are no absorption lines. The object appeared to be embedded in bright $\mathrm{H}$ 
II regions near the nucleus of the distorted SBbc galaxy NGC $4618(v=532 \mathrm{~km}$ $\mathrm{s}^{-1}$ ), and by December 1985 it had faded so much that only the very strongest emission lines could be detected. There is no doubt that the object was a supernova, but at the time of discovery its absolute brightness $\left(M_{V} \approx-14\right.$, or -15 if $A_{V}=1 \mathrm{mag}$ ) was much smaller than that of SNe I, SNe I-pec, or SNe II at maximum $\left(M_{V} \approx-18\right.$ to -20$)$.

Most of the emission lines in SN $1985 \mathrm{f}$ have now been identified (Filippenko and Sargent 1986a), but in some cases the blends are difficult to decompose in an objective manner. The strongest feature is $[\mathrm{O} \mathrm{I}] \lambda \lambda 6300,6364$, followed by [Ca II] $\lambda \lambda 7292,7324$. Other lines include a blend of O I, Ca II, N I, and [C I] at $\sim \lambda 8700,[C$ I] $\lambda \lambda 9823,9849, O$ I $\lambda 7774, \mathrm{Na}$ I $\lambda \lambda 5890,5896$, and $\mathrm{Mg}$ I] $\lambda 4571$. A very broad bump is apparent between $\sim \lambda 3800$ and $\lambda 5800$, especially in a plot of $f_{\lambda}$ versus $\lambda$ (Filippenko and Sargent 1986a). If many multiplets of Fe II produce this feature, then the strength of iron lines is comparable to that

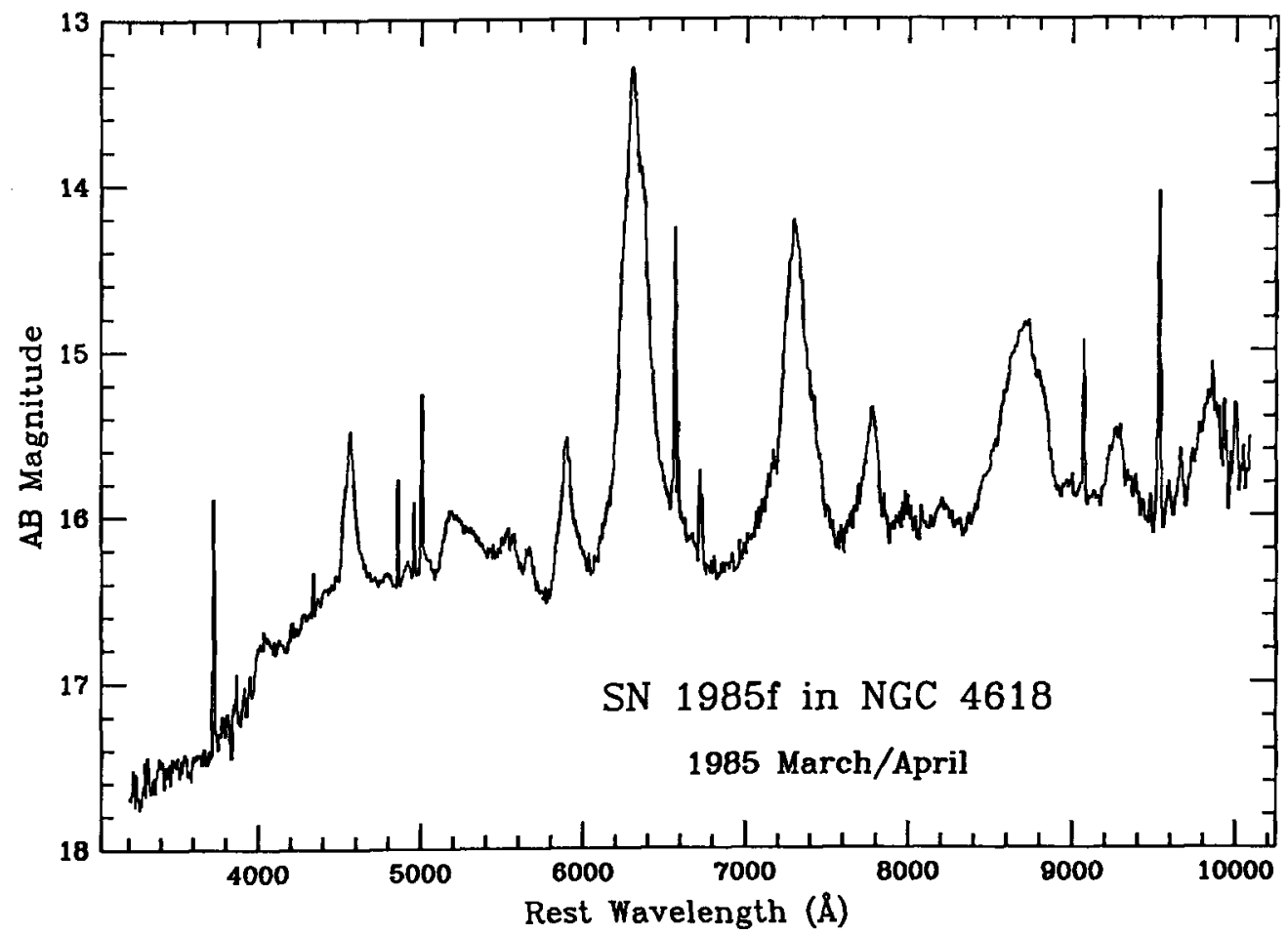

Figure 3: A composite of several spectra of SN 1985f in NGC 4618, obtained in March and April 1985 with the Palomar 5-m and Lick 3-m reflectors. Broad emission lines are produced by the $\mathrm{SN}$, whereas narrow lines come from the surrounding $\mathrm{H}$ II regions. Relative intensities of narrow lines separated by more than $\sim 2000 \AA$ may be erroneous because the entrance aperture was not of uniform size during the various observations. The spectrum differs radically from those in Figs. 1 and 2, and does not resemble spectra of normal SNe I or SNe II at any evolutionary stage. It is, however, similar to the late-time spectra of $\mathrm{SNe}$ I-pec. 
of oxygen. Lines of $\mathrm{H}$ and $\mathrm{He}$ are conspicuously absent. Although most of the features are produced by transitions between the lowest energy levels of neutral atoms, the presence of $\mathrm{Fe}^{+}, \mathrm{Ca}^{+}$, and lines from some upper levels of $\mathrm{O}$ suggest that Balmer emission should be visible unless $H$ is severely underabundant. $A$ similar, but less definite, conclusion can be drawn for He.

The optical spectrum in Figure 3 bears absolutely no resemblance to those of SNe I (Fig. 1) or SNe II (Fig. 2) within a month past maximum brightness, nor is it similar to SNe I-pec near maximum. A search through the literature (e.g., Zwicky 1965; Greenstein and Minkowski 1973; Kirshner et al. 1973; Oke and Searle 1974, and references therein) shows that no normal SNe I or SNe II have been found which exhibit the spectral characteristics of SN 1985f, even long after maximum brightness. In addition, Zwicky's (1965) Types III, IV, and V differ greatly from SN $1985 \mathrm{f}$.

Filippenko and Sargent $(1985,1986 a)$ pointed out that SN $1985 f$ may be the progenitor of supernova remnants (SNRs) such as Cas A (Kirshner and Chevalier 1977), N132D in the LMC (Lasker 1978), G292.0 + 1.8 in our Galaxy (Goss et al. 1980), and SB 187 in NGC 4449 (Balick and Heckman 1978; Kirshner and Blair 1980; Blair, Kirshner, and Winkler 1983). These objects all have extraordinary elemental abundances; in particular, some filaments in Cas A consist largely of oxygen. Simple calculations demonstrate that the ejecta of SN $1985 \mathrm{f}$ contain at least $1 M_{\odot}$ of $\mathrm{O}$, and some estimates go as high as $5 M_{\odot}$ (Begelman and Sarazin 1986). Chevalier (1976) suggested that the progenitor star of Cas A lost much of its outer atmosphere of $\mathrm{H}$ prior to exploding, and therefore was subluminous. The absence of $\mathrm{H}$ in the spectra of the rapidly-moving ejecta is also explained by this scenario. If $\mathrm{SN} 1985 \mathrm{f}$ was near maximum when first detected in February 1985 , then it too was much fainter than normal SNe, and a close connection to Cas A remnants is more likely. These ideas are reinforced by the calculations of Cahen, Schaeffer, and Cassé (1986).

Although the spectrum of SN $1985 \mathrm{f}$ was undeniably "unique" at the time of discovery, several recent observations show that the late-time ( $t \gtrsim 8$ months) spectra of SNe I-pec are virtually identical to that in Figure 3 (Kirshner 1985, quoted in Chevalier 1986; Gaskell et al. 1986). These exciting results imply that SN 1985 f was an "old" SN I-pec in February 1985. It is unfortunate that the object was not discovered earlier, since it would have been extremely bright $(m \approx 12-12.5)$ at maximum.

The connection between SN 1985f and SNe I-pec strongly supports the view that these objects represent a fundamentally new class of supernovae whose progenitors are massive Wolf-Rayet stars that lost their outer layers of $H$. They come from a different stellar population than normal SNe I, but they are also quite different from SNe II in their physical properties. Since Zwicky's (1965) Types III, IV, and possibly V SNe may simply be peculiar SNe II (Oke and Searle 1974; DB85), it is proposed that SNe I-pec be renamed as Type III objects (see also Chevalier 1986). Further study of their properties should be exceptionally rewarding.

\section{ACKNOWLEDGMENTS}

Expert assistance with telescopes and equipment was provided by the day and night crews at Lick and Palomar Observatories. The spectra in Figure 1 are published with the permission of A. C. Porter. I thank R. A. Chevalier, A. C. 
Porter, and W. L. W. Sargent for illuminating discussions. Financial support was received from the Miller Institute for Basic Research in Science (U.C.B.), and from the International Astronomical Union.

\section{REFERENCES}

Axelrod, T. S. 1980, in Type I Supernovae, ed. J. C. Wheeler (Austin: University of Texas), p. 80.

Balick, B., and Heckman, T. 1978, Ap. J. (Letters), 226, L7.

Barbon, R. 1978, Mem. Soc. Ast. It., 49, 331.

Barbon, R., Ciatti, F., and Rosino, L. 1973, Astr. Ap., 25, 241.

Barbon, R., Ciatti, F., and Rosino, L. 1979, Astr. Ap., 72, 287.

Begelman, M. C., and Sarazin, C. L. 1986, Ap. J. (Letters), in press.

Bertola, F. 1964, Ann. d'Ap., 27, 319.

Bertola, F., Mammano, A., and Perinotto, M. 1965, Contr. Asiago Obs., No. 174.

Blair, W. P., Kirshner, R. P., and Winkler, P. F., Jr. 1983, Ap. J., $272,84$.

Branch, D. 1982, in Supernovae: A Survey of Current Research, eds. M. J. Rees and R. J. Stoneham (Dordrecht: Reidel), p. 267.

Branch, D., Falk, S. W., McCall, M. L., Rybski, P., Uomoto, A. K., and Wills, B. J. 1981, Ap. J., 244, 780.

Branch, D., Lacy, C. H., McCall, M. L., Sutherland, P. G., Uomoto, A., Wheeler, J. C., and Wills, B. J. 1983, Ap. J., 270, 123.

Cahen, S., Schaeffer, R., and Cassé, M. 1986, in Fifth Moriond Astrophysics Meeting: Nucleosynthesis and its Implications on Nuclear and Particle Physics, ed. J. Audouze (Dordrecht: Reidel).

Chevalier, R. A. 1976, Ap. J., 208, 826.

Chevalier, R. A. 1986, Highlights of Astronomy, this volume.

De Robertis, M. M., and Pinto, P. A. 1985, Ap. J. Letters, 293, L77.

Doggett, J. B., and Branch, D. 1985, A. J., 90, 2303 (DB85).

Elias, J. H., Matthews, K., Neugebauer, G., and Persson, S. E. 1985, Ap. J., $296,379$.

Filippenko, A. V., and Sargent, W. L. W. 1985, Nature, 316, 407.

Filippenko, A. V., and Sargent, W. L. W. 1986a, A. J., in press (April issue).

Filippenko, A. V., and Sargent, W. L. W. 1986b, in preparation.

Gaskell, C. M., Cappellaro, E., Dinerstein, H., Garnett, D., Harkness, R. P., and Wheeler, J. C. 1986, Ap. J. (Letters), in press.

Goss, W. M., Ekers, R. D., Danziger, I. J., and Israel, F. P. 1980, M. N. R. A. $S ., 193,901$.

Greenstein, J., and Minkowski, R. 1973, Ap. J., 182, 225.

Kirshner, R. P. 1985, private communication.

Kirshner, R. P., and Blair, W. P. 1980, Ap. J., 236, 135.

Kirshner, R. P., and Chevalier, R. A. 1977, Ap. J., 218, 142.

Kirshner, R. P., and Kwan, J. 1974, Ap. J., 193, 27.

Kirshner, R. P., and Kwan, J. 1975, Ap. J., 197, 415.

Kirshner, R. P., Oke, J. B., Penston, M. V., and Searle, L. 1973, Ap. J., 185, 303.

Lasker, B. M. 1978, Ap. J., 223, 109.

Litvinova, I. Yu., and Nadyozhin, D. K. 1983, Ap. Space Sci., 89, 89.

Minkowski, R. 1939, Ap. J., 89, 156. 
Niemela, V. S., Ruíz, M. T., and Phillips, M. M. 1985, Ap. J., 289, 52.

Oke, J. B., and Searle, L. 1974, Ann. Rev. Astr. Ap., 12, 315.

Panagia, N. 1986, in High Energy Phenomena Around Collapsed Stars (Cambridge: Cambridge University Press), in press.

Porter, A. C. 1983, Bull. Amer. Astr. Soc., 15, 966.

Schurmann, S. R., Arnett, W. D., and Falk, S. W. 1979, Ap. J., 230, 11.

Uomoto, A., and Kirshner, R. P. 1985, Astr. Ap., 149, L7.

Wheeler, J. C., and Levreault, R. 1985, Ap. J. (Letters), 294, L17.

Zwicky, F. 1965, in Stars and Stellar Systems, Vol. 8, eds. L. H. Aller and D. B. McLaughlin (Chicago: University of Chicago Press), p. 367. 